\title{
Study of the parameters and operating modes of the installation for aerosol treatment of seed grain
}

\author{
Vladimir Teterin ${ }^{1}$, Vyacheslav Terentyev ${ }^{2}$, Konstantin Andreev ${ }^{2,}$, Alexander Shemyakin ${ }^{2}$, \\ and Olga Teterina ${ }^{2}$ \\ ${ }^{1}$ Federal Scientific Agro-Engineering Center VIM, Moscow, Russia \\ ${ }^{2}$ Ryazan State Agrotechnological University Named after P.A. Kostychev, Ryazan, Russia
}

\begin{abstract}
One of the modern elements of agrotechnologies is the presowing treatment of seeds with bio-stimulating preparations, providing a complex effect on seeds, which, along with improving the sowing and productive qualities of seeds, can increase the resistance of plants to adverse environmental factors, diseases and pests. Recently, world agricultural production is increasingly focused on environmentally friendly technologies, where preparations based on components of natural origin become important. Preparations based on humic acids are among them. The effectiveness of their use in the process of presowing treatment of seeds to a certain extent depends on the technology and technical means used. This article discusses the design of the developed seed dresser and explores the basic physical processes that affect the quality of seed treatment. The effectiveness of seed treatment with growth stimulants has been studied depending on the intensity of heat exchange processes taking place in the processing chamber of the developed device, where heat transfer from the vapor of humates to the downward flow of grain occurs. Herewith, the dependence of heat transfer on the residence time of the grain in the treatment chamber, heat carrier temperature (aerosol of humates) and uniform distribution of coolant flow in the treatment chamber was determined.
\end{abstract}

\section{Introduction}

The main indicators in the production of grain crops are the yield and quality parameters of the resulting products, which primarily depends on agricultural technologies and physiological processes occurring during the growth and development of plants.

One of the important elements of agricultural technologies that affect this parameter is the pre-sowing treatment of seed material, suggesting a complex effect on seeds, contributing to an increase in sowing and productive qualities of seeds, plant resistance to adverse environmental factors, diseases and pests. Nowadays, plant growth stimulators of various nature are becoming more common for the presowing treatment of seed material,

*Corresponding author: kosta066@yandex.ru 
while preparations that are based on components of natural origin are becoming more and more important, which is associated with high demand for environmentally safe products $[2,3,6]$.

One of them are preparations based on humic acids. Humic acids are a class of compounds formed as a result of the decomposition of organic substances, in particular plant fiber. They are natural components of drinking water, soil and brown coal [7]. According to a number of scientists, the treatment with humic preparations weakens the negative value of damages of plant seeds and improves the sowing qualities of seeds: laboratory and field germination of seeds, viability, stimulates the growth and development of seedlings and the primary root system, while reducing seed damage by fungal diseases that have arisen in result of internal seed infection [1, 4]. In this connection, these preparations can be used in the process of presowing treatment of seed material, as well as plant growth stimulants for growing most crops.

The effectiveness of growth stimulants, including preparations based on humic acids in the process of presowing treatment to a certain extent depends on the technology and technical means used in its implementation. Currently, seed dressers with a dispersion of drops from 30 to 120 microns are mainly used. Reducing the dispersion of droplets, active mixing of seeds, as well as heat exposure can enhance the effect of the use of preparations based on humic acids in the process of presowing treatment of seeds. For this purpose, a hot mist seed treatment device was developed (Fig. 1).

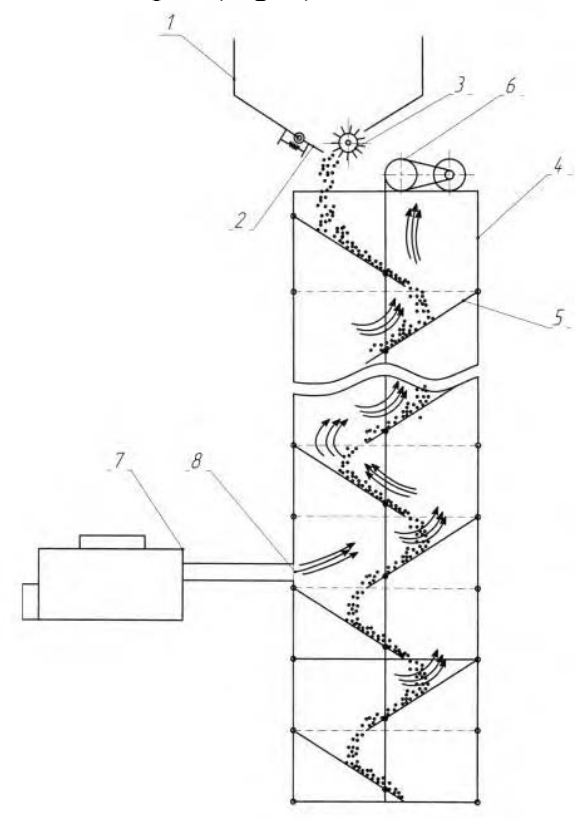

Fig. 1 Diagram of the device for the aerosol treatment of seed grain with protective stimulating substances. 1 - seed tanker; 2 - metering device with ribbed drive roller; 3 - movable bottom; 4 treatment chamber; 5 - inclined shelves; 6 - cable-drum mechanism; 7 - aerosol generator; 8 - nozzle.

The device for aerosol treatment of grain crops consists of a seed tanker, a metering device with a ribbed drive roller and a movable bottom, a treatment chamber with inclined shelves and a cable drum mechanism, a hot mist generator with a nozzle.

The device works as follows. Seeds by gravity from the tanker fall into the space between the ribbed drive roller and the adjustable movable bottom. Then, the dosed seeds enter the treatment chamber and are sent along inclined shelves, forming a downward flow. The hot mist generator delivers an aerosol of protective-stimulating substances with a 
temperature of $50-60^{\circ} \mathrm{C}$ to the treatment chamber, where it mixes with the seed flow. Due to repeated contact of the aerosol with the seeds when seeds are poured from the shelf to the shelf and uniform mixing, the treatment efficiency increases. As a result of more than $30^{\circ}$ $\mathrm{C}$ temperature difference between cold seeds and an aerosol, the phase transition of the aerosol to the liquid on the surface of the seed occurs, which contributes to a thin uniform film on the surface of the seed. The increased temperature of the aerosol provides heat treatment of the seeds and contributes to the activation of the physiological processes of the grain. The movement of the warm aerosol stream in the upper zone of the treatment chamber forms a stream that provides air suction in the upper zone of the chamber.

Dosing of seeds can be carried out as a change in the frequency of rotation of the ribbed roller, and a change in the gap between the ribbed roller and the movable bottom. In addition, the movable bottom ensures the tanker discharge in case of breakdowns. Inclined shelves are connected to a cable-drum mechanism, which adjusts the tilt angle of the shelves, thereby allowing to change the speed of movement of the seeds in the treatment chamber [5].

\section{Materials and methods}

The effectiveness of seed treatment with growth stimulants depends on the intensity of heat exchange processes taking place in the treatment chamber of the plant, where heat transfer from the steam of humates to the downward flow of grain occurs, while the heat transfer depends on the residence time of the grain in the treatment chamber, heat carrier temperature (aerosol of humates), and uniformity distribution of the coolant flow in the treatment chamber.

The time spent by the grain in the treatment chamber will primarily depend on the speed of its movement along the inclined shelves, which is determined by the tilt angle of the shelf and the initial position of the grain on the shelf at the moment of the motion start.

To eliminate the influence of random factors on the process of grain movement on a metal plate, the tilt angle of which was set using a pendulum type protractor of Zuri-M brand, the grain was poured onto the plate and held with the help of another plate at the desired level (initial position). Then the plate was sharply removed, so that the initial grain velocity in the experiment was absent. The whole process of grain movement was filmed on a high-speed camera in high quality mode, the shots were subjected to additional decoding in the program for professional video processing VegasPro 13.0. The reliability of the data obtained was evaluated using the Fisher criterion. Wheat grain variety "Helios" with $14 \%$ moisture was used as the material for the study.

The quality of seed treatment also depends on the uniform distribution of the coolant flow in the treatment chamber. To determine this parameter, beacons with high thermal inertia were installed in a certain way inside the treatment chamber. They were installed on a grid made of a thread with low thermal conductivity, which in turn was attached to a special frame. The resulting design could be quickly removed and mounted inside the treatment chamber. Frames were installed across the entire width of the treatment chamber, forming a coordinate space for determining the temperature at different points in the treatment chamber.

As a result of reading and location of temperature points in space, a topographical picture of the temperature field was formed, that is, the topography of the distribution of heat flows in the treatment chamber. The layout of the beacons in the treatment chamber is shown in Fig. 2. 


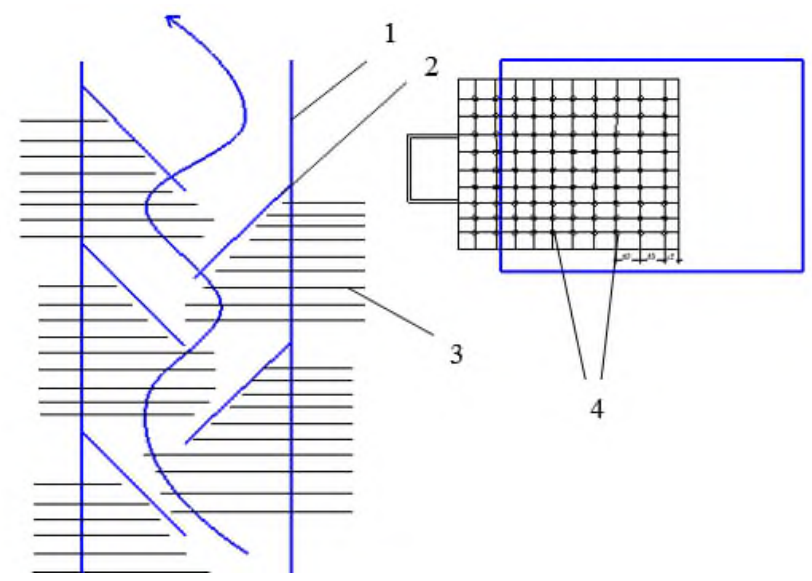

Fig. 2 The layout of the beacons in the treatment chamber. 1 - treatment chamber, 2 - treatment chamber shelves, 3 - frames, 4 - temperature beacons.

The treatment chamber was heated by hot mist for 5 minutes and reached steady-state operation, then each of the grids was quickly removed and photographed using RGKTL-80 thermal imager.

The aerosol was fed using BF-150 hot mist generator. In the maximum aerosol temperature mode, the grids were installed above and below the inclined shelf to assess the uniform distribution of heat flows across the width of the treatment chamber. As a result of the study, it was found that the heating of thermal beacons reached a temperature of 21-22 ${ }^{\circ} \mathrm{C}$ with an average ambient temperature of about $19^{\circ} \mathrm{C}$. The ambient air humidity was about $95 \%$.

The experimental studies also took into account the initial temperature of the aerosol of protective-stimulating substances, the consumption of fuel and working fluid, the speed of the upward flow of the aerosol of protective-stimulating substances and the size of the treatment chamber. The results of the experiment were processed using the method of mathematical statistics and three-dimensional geometry.

\section{Results and discussion}

Analysis of studies of the processes of grain movement showed that the speed of grain increases with increasing the tilt angle of the shelf relative to the horizontal. The initial position of the grain on the shelf has a significantly less effect on speed than the change in the tilt angle.

As a result of investigations, experimental data were obtained, on the basis of which an empirical dependence was built:

$$
\mathrm{V} 3=-1.9101+0.1578 * \mathrm{x}+0.9404 * \mathrm{y}-0.0016^{*} \mathrm{x} 8 \mathrm{x}-0.1475^{*} \mathrm{x} * \mathrm{y}+1.2052 * \mathrm{y} * \mathrm{y}
$$

where $\mathrm{V} 3$ is grain velocity, $\mathrm{m} / \mathrm{s}$;

$\mathrm{x}$ is the tilt angle of the shelf, degrees;

$\mathrm{y}$ is the initial position of the grain, $\mathrm{m}$.

The adequacy of this mathematical model to the experimental data was expressed by the coefficient of determination, which amounted to 0.888 .

The plot based on the empirical dependence is shown in Fig. 3. 


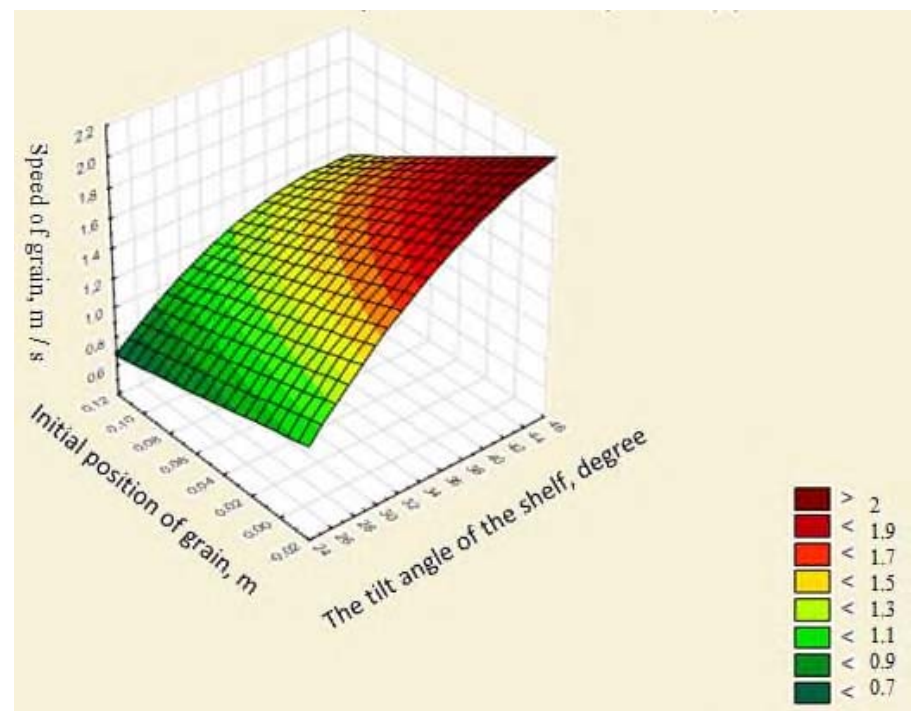

Fig. 3 Dependences of the grain velocity on the tilt angle of the shelf and its initial position.

The convergence of experimental data with theoretical studies was $5 \%$. Analysis of the experimental data made it possible to establish that the effect of the initial position of the grain on the shelf has a significantly lower value on the speed of movement of the grain than the tilt angle of the shelf, therefore, to adjust the rational speed of the grain movement in the treatment chamber, an adjustment of the tilt angle of the shelves should be provided. The distance between the shelves should be chosen based on considerations of limiting the speed of collision when pouring grain from the shelf to the shelf, as well as ensuring the least resistance to movement of the upward flow of the aerosol.

The distribution of heat flows was carried out with different loading of the grain treatment chamber. The topography of the thermal field was determined at different levels of the treatment chamber.

On the basis of measuring the temperature of the beacons, it was found that the efficiency of the distribution of the aerosol across the width of the treatment chamber is from 87 to $94 \%$, the pattern of the distribution of the thermal field, and hence the aerosol, is seen in Fig. $s 4$ and 5.

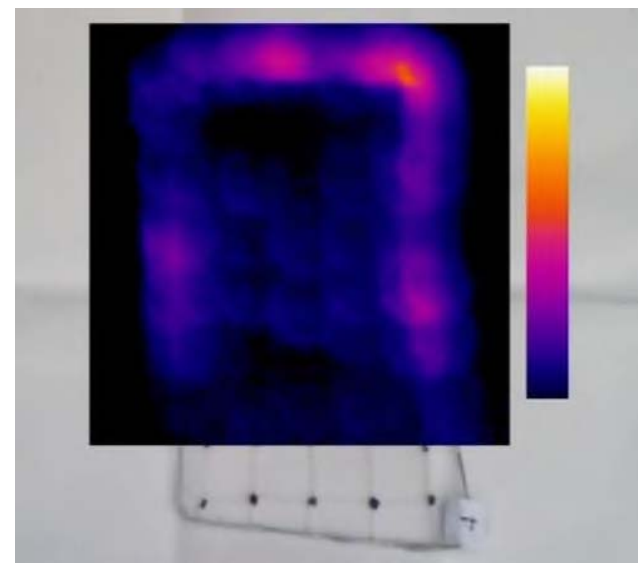

Fig. 4. Beacon temperature distribution within the frame at level 4 


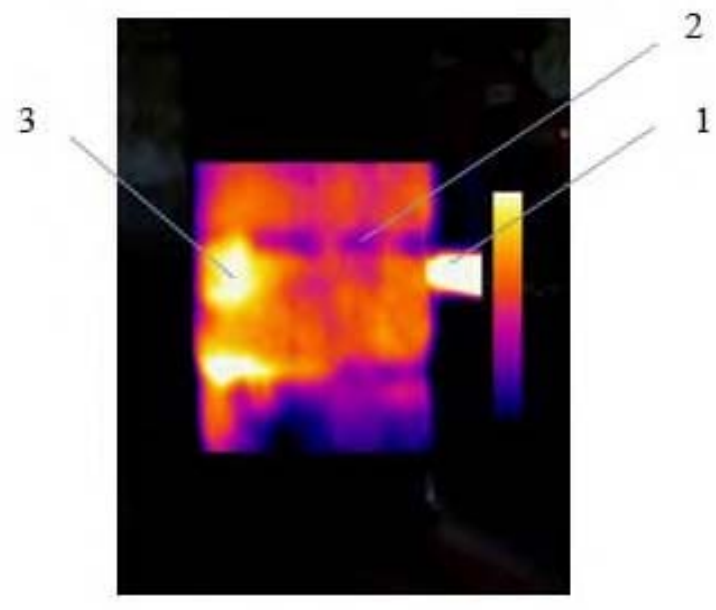

Fig. 5. Heat pattern of the treatment chamber of the device for seeds treatment with growth stimulants, obtained by RGKTL-80 thermal imager. 1 - hot mist generator nozzle; 2 - location of shelves in the treatment chamber; 3 - zone of maximum heating of the treatment chamber.

Analysis of the temperature distribution shows that the aerosol is fairly evenly distributed within the treatment chamber, which suggests that the treatment of seeds with the aerosol with protective-stimulating substances occurs evenly, and as a result of the seeds treatment, both the uniformity of the aerosol and the seeds heating will be ensured.

\section{Conclusion}

The use of the device for seed dressing makes possible effective presowing aerosol treatment of seeds with protective-stimulating substances as a result of combined heat and some preparative effect. Studies have shown that a change in the tilt angle from 250 to 400 degrees leads to an increase in speed in the range from 0.8 to $1.8 \mathrm{~m} / \mathrm{s}$ and affects the performance of the plant and the quality of seed treatment. As a result of the study, it was found that the heating of thermal beacons reached a temperature of $21-22{ }^{\circ} \mathrm{C}$ with an average ambient temperature of about $19^{\circ} \mathrm{C}$. The efficiency of aerosol distribution across the width of the treatment chamber was from 87 to $94 \%$, which was confirmed by the pattern of the thermal field distribution. The use of the developed device for aerosol treatment of seed grain will reduce the energy costs of mixing the seeds during the processing and increase the treatment efficiency through the use of fine aerosol of protective-stimulating substances. In addition, the device has high reliability due to the small number of moving parts.

\section{References}

1. X. Chen The use of humic acid urea fertilizer for increasing yield and utilization of nitrogen in sweet potato. Plant, Soil and Environment 63(5), 201-206 (2017)

2. A. Da Piedade Melo et al., Mixed rhizobia and Herbaspirillumseropedicae inoculations with humic acid-like substances improve water-stress recovery in common beans. Chemical and Biological Technologies in Agriculture 4(1), 6 (2017) 
3. L.H. Dos Santos Carbon of humic substances in soil aggregates cultivated with onion under no-till and conventional tillage systems. Idesia 36(1), 15-25 (2018)

4. I.M Nikitina Humic acids of solid fossil fuels-perspectives for application in technology and environment protection. Eurasian Mining, 2, 33-36 (2016)

5. N.V. Byshov, S.N. Borychev, O.A. Teterina, M.Yu. Kostenko, G.K. Rembalovich, R.V. Beznosyuk, V.S. Teterin; Patent 2682885 Russian Federation, IPC: A01C 1/06. Device for seed dressing. applicant and patent holder FSBEI HE RSATU. no. 2682885; decl. 02.22.2018; publ. 03/22/2019. Byul. no. 9.

6. R. Pei Effects of application of humic acid on yield, nitrogen use efficiency of summer maize. Scientia Agricultura Sinica, 50(11), 2189-2198 (2017)

7. Q. Sun Different chemical activation of humic acid from weathered coal affect the growth and development of tomatoes and cabbages. IOP Conference Series: Earth and Environmental Science, 170(2) (2018) 